\title{
Environmental Effects On Phase Coherent Underwater Acoustic Communications: A Perspective From Several Experimental Measurements
}

\author{
T. C. Yang, Naval Research Lab., Washington DC 20375
}

\begin{abstract}
This paper presents a summary of results from several experiments using phase coherent underwater acoustic communications in shallow waters with weak to strong internal waves. Measurements of temporal coherence time of the underwater acoustic channels are presented and related to the equalizer performance.
\end{abstract}

\section{INTRODUCTION}

Phase coherent communications provide a high data rate for a given bandwidth (compared with phase incoherent communications) and are particularly useful for wireless communications in a band-limited underwater acoustic channel. Successes of phase coherent underwater acoustic communications (ACOMMS) have been recently demonstrated after the introduction of adaptive channel decision feedback equalizer (DFE) in combination with the phase locked loop (PLL) [1]. The problem, however, is the reliability of the equalizer in terms of bit error rate (BER) over a large number of packets. Typically, about a fraction (e.g., one third) of the packets are error-free before error decoding. The unreliability is largely attributed to the "catastrophic" error propagation problem of the DFE. As symbol errors occur when the tap coefficients of the equalizer are incorrectly estimated, the errors propagate to later symbols through the use of incorrect tap coefficients (before they are updated/corrected). It is generally noted that the performance of DFE is sensitive to the channel condition, particularly the temporal variation of the channel. The question is then how is BER affected by the channel condition.

To track the channel variation and counter the error propagation, the equalizer is adaptively updated using the training or decision symbols (during the training and message data phases). One finds empirically that the DFE is updated for more than $99 \%$ of the time (or symbols) even for successfully transmitted data packages. This has led to the suggestion that the acoustic communication channel is rapidly varying at the time scale of symbols. Is the BER related to the rate of temporal variation?

The purpose of this paper is to investigate the rate of temporal variation of the underwater acoustic channel and how it affects ACOMMS. If the channel is indeed 
changing at the symbol rate, then the physical mechanism of what causes such rapid channel changes needs to be understood. To investigate the channel fluctuation rate, we conducted several experiments in shallow water environments with weak to strong internal wave activities. We use consecutive m-sequences to measure the channel impulse response functions at the scale of tens to hundreds of symbols. The motivation was that if the channel were rapidly changing at the scale of symbols, we would definitely observe changes in the channel impulse response functions at the scale of tens to hundreds of symbols.

The result of this investigation is reported here based on measurements from six experiments at different frequencies and different locations. We find that in general, the channel impulse response functions change rapidly from packet to packets (usually separated by a few minutes). But what is relevant for ACOMMS is not the inter-packet coherence but the intra-packet coherence. We find that at mid-frequencies, most of the data packets have intra-packet coherence $>0.9$ indicating that the channel is very stable within a packet. However, the symbols do incur an extra phase, which changes rapidly at the scale of a few symbols. We find that the decision feedback equalizer (DFE/PLL) in these cases still shows a high $(>99 \%)$ update rate, the reason being that both the DFE and PLL try to compensate for the rapid symbol phase change. We determine that the high update rate is an artifact of the processor and does not reflect the true condition of the channel. The channel can be equalized with almost no update using a different approach.

\section{TIME SCALE OF THE OCEANOGRAPHIC PROCESSES}

Shallow (coastal) water is known for its complexities. The effect of the random medium on acoustic signal propagation can be analyzed by the time scale of the various oceanographic processes. The intrusion of fresh water and eddies turns the coastal shallow water into a range dependent environment for signal propagation. These processes are slowly varying and are generally stable over a time scale of tens of minutes. The internal waves have a time scale of $\sim 1-10$ minutes. The turbulence, rough sea surfaces etc. can have a much shorter time scale (seconds). How the internal waves affect signal propagation has been a subject of great interest recently. Several experiments have been conducted in the last 10 years to study the effect of the internal waves on signal propagation at low frequencies, as reported in the open literature. At low $(<1 \mathrm{kHz})$ frequencies, it has been reported that the signal characteristics change drastically at the scale of $\sim 2$ minutes. The most noticeable changes include the depth where the signal intensity peaks, the multipath arrival (time) pattern, and the signal amplitude and phase as a function of depth. The changes are more pronounced when solitary internal waves are present, which cause significant mode coupling and consequently the change of the mode amplitudes (of the signals) with respect to range. These changes are important for low frequency $(<1 \mathrm{kHz})$ ACOMMS since the packet length is of the order of 1-2 minutes for a data packet of 5-10 kbits.

At mid $(2-5 \mathrm{kHz})$ to high $(>15 \mathrm{kHz})$ frequencies, the internal waves are not expected to have any significant effect on ACOMMS since the packet length is $<5-10 \mathrm{sec}$. At this time scale the internal waves can be treated as a deterministic (range dependent) 
environment. However, the internal waves can cause severe signal fading, which is a signal-to-noise ratio problem. At mid and high frequencies, the turbulence and sea surface waves are believed to have a (more) significant effect on signal propagation. The effects of these processes on signals are not yet completely characterized (quantitatively). For a downward refractive sound speed profile, the sea surface effect is less significant at lower frequencies, particularly if the source and receiver are in the duct.

What physical processes can cause channel variation at the symbol rate? The answer depends on the carrier frequency. Let us consider, for example, ACOMMS with a bandwidth of $500 \mathrm{~Hz}$. Each symbol is $2 \mathrm{msec}$ long. At a (carrier) frequency of $20 \mathrm{kHz}$, the micro and fine structure of the ocean medium has a spatial scale comparable to the acoustic wavelength, and therefore could easily affect the acoustic signals and produce temporal variations at the scale of $\sim 10 \mathrm{msec}$ ( 5 symbols). At mid frequencies, the ocean inhomogeneities that have a scale comparable to the acoustic wavelength include the turbulence, and high wavenumber spectral components of the internal waves. Most of these processes have a temporal scale of seconds. At low frequencies, the dominant oceanic process is likely to be the internal waves, which have a temporal scale of minutes. Other processes such as ocean fronts, eddies, and intrusion of fresh water have a temporal scale of hours. Hence, at low and mid frequencies, it seems unlikely that any of the above oceanographic processes would cause fluctuation at the scale of $\sim 10 \mathrm{msec}$ ( 5 symbols) or less.

The above issues prompted us to conduct a systematic measurement of the channel coherence as a function of time at different frequencies.

\section{EXPERIMENTAL MEASUREMENTS}

To measure the environmental impact on ACOMMS, data were collected in dynamic shallow water using a fixed source and fixed receivers. To measure the effect of platform motion, data were collected in a relatively stable environment using a moving source and fixed receivers. The rate of the channel fluctuation can be characterized by the signal Doppler spread or the temporal coherence time.

The results of six experiments are reported here. Advent 99 was conducted by NURC (previously SACLANTCEN) on the Adventure bank west of Sicily in the Mediterranean $[2,3]$. Water depth was $\sim 80 \mathrm{~m}$. The source-receiver range is $10 \mathrm{~km}$. Conductivity, temperature and Depth (CTD) data indicated the presence of weak internal waves. The ASCOT01 experiment was also conducted by NURC in the Massachusetts Bay east of Boston along the contour of $100 \mathrm{~m} \mathrm{[4].} \mathrm{The} \mathrm{same} \mathrm{source-receiver} \mathrm{geometry} \mathrm{was} \mathrm{used.}$ This area is known to have non-negligible internal wave activities. The SWARM95 and RAGS03 experiments were conducted off the New Jersey coast in water of 40-80 m depth using a fixed source and VLA receivers [5]. The source to NRL array range was $40 \mathrm{~km}$ in SWARM 95 and $3 \mathrm{~km}$ in RAGS03. Strong internal wave activities were reported [5]. The RDS3 experiment was conducted in the Adriatic Sea off the coast of Bali, Italy. The water depth was $\sim 110 \mathrm{~m}$. CTD data indicated a stable sound speed profile over a period of hours. The MREA03 experiment was conducted north of Elba in the Mediterranean. The water depth was $\sim 88 \mathrm{~m}$. The sound speed profile was stable over 
a period of hours. Both RDS3 and MREA03 experiments used a towed source and a fixed VLA.

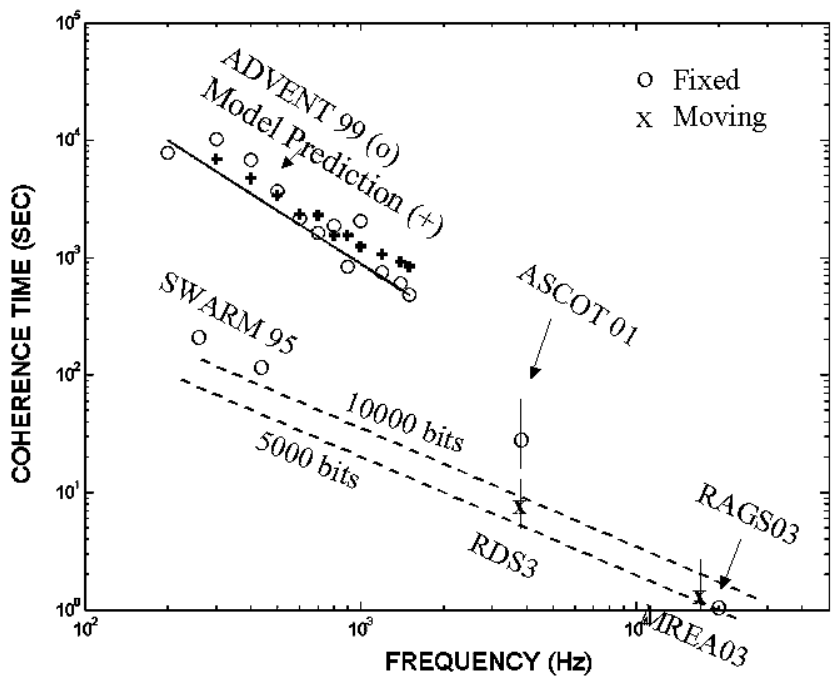

FIGURE 1. Temporal coherence time as a function of frequency from six experiments. The dashed lines indicate the packet duration as a function of the carrier frequency for 5 and $10 \mathrm{kbits}$ of data respectively (see text). The vertical bar indicates the variation of the coherence time over the data samples.

For the temporal coherence measurements, multitone data in the band of 200-1,500 $\mathrm{Hz}$ were used for the Advent99 exp. The rest of the experiments used consecutive m-sequences, which led to measurements of channel impulse response functions at the scale of hundreds of msec. Temporal coherence is defined as the cross correlations of an initial signal vector with a later signal vector normalized by the norm of the two vectors. Temporal coherence time is defined as the time when the temporal coherence between the two signals drops to 0.8 . We use 0.8 instead of the standard 0.5 because (1) temporal coherence usually does not follow an exponential dependence as normally assumed, and (2) acoustic communication normally requires high $(>0.8)$ coherence.

Figure 1 shows the measured temporal coherence time from the various experiments. The coherence time measurements for the Advent 99, ASCOT01, RDS3 and SWARM99 experiments have been reported previously [2,3,6,7].

Also shown in Fig. 1 are two dashed lines which show the communication packet duration (length) as a function of the carrier frequency for data packets of 5 and 10 kbits respectively. We assume binary phase-shifted keying signal with a bandwidth one quarter of the carrier frequency. In general, we want the packet length to be less than or equal to the channel coherence time, beyond which the signal becomes less coherent. We note that for the ASCOT01 environment, the channel can support packet transmission of up to 20 kbits data (lasting $20 \mathrm{sec}$ ), but for RDS3 experiment, the channel can only support data packets of 5-10 kbits. As shown in Fig. 1, the packet length at low $(<1 \mathrm{kHz})$ frequencies is rather long due to the limited bandwidth. When the packet length approaches the channel coherence time as is the case for the SWARM 95 environment, appreciable variation in the impulse response function will impede the equalizer performance. One finds that the data are successfully equalized (BER is $<1 \%$ ) only for those (portions of the) packets with high $(>0.7-0.8)$ intra-packet coherence. Figure 1 also shows that at high $(>15 \mathrm{kHz})$ frequencies, the channel coherence time is rather short allowing only short packets to be transmitted successfully. 


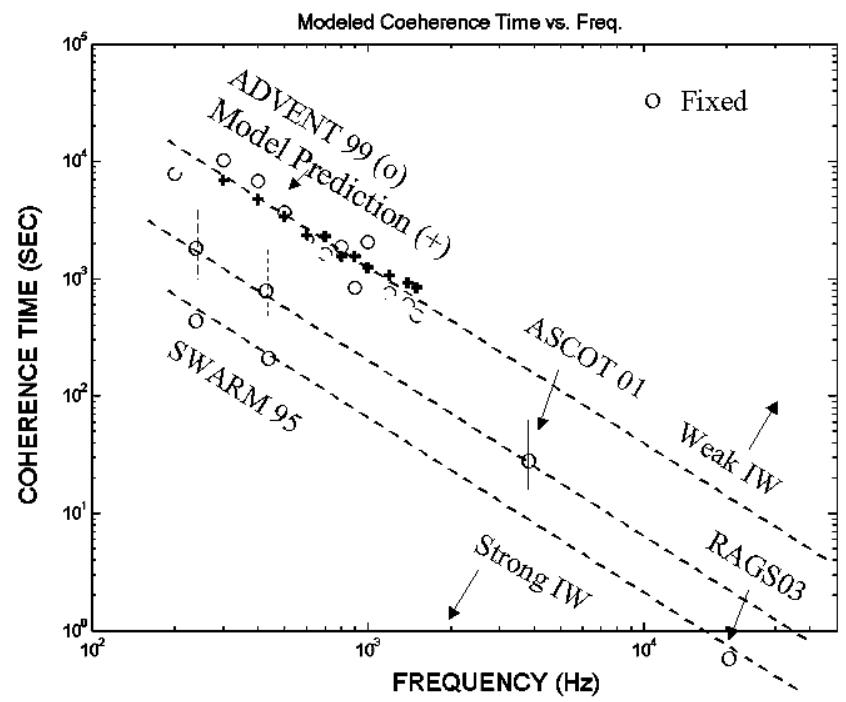

FIGURE 2. Temporal coherence time scaled to a range of $10 \mathrm{~km}$. The dashed lines indicate -1.5 power of frequency dependence. The data are separated into weak, intermediate and strong internal wave regions.

We note that the temporal coherence time is shorter for a moving source than a stationary source. The temporal coherence time for a moving source is given by the horizontal coherence length divided by the platform speed, which is often smaller than the (intrinsic) temporal coherence time associated with the medium. Compare RDS3 data with the ASCOT01 data.

Internal waves seem to also reduce the channel coherence time. Figure 2 shows the channel coherence time using only fixed source-receiver data. The coherence time in Fig. 2 is scaled to a fixed range of $10 \mathrm{~km}$ assuming the coherence time is inversely proportional to the square-root of range. Figure 2 indicates that the coherence time $\tau$ can be divided into regimes of weak, intermediate and strong internal waves with coherence time given by

$$
\tau \sim \alpha \mathrm{R}^{1 / 2} \mathrm{f}-1.5
$$

with $\alpha(10 \mathrm{~km})=(0.25-4) \cdot 10^{5}(\text { meter sec })^{1 / 2}$.

\section{PERFORMANCE ANALYSIS}

For performance analysis, we shall concentrate on the ASCOT01 data and illustrate the environmental influence [6]. We plot in Fig. 3 the channel impulse response functions at the scale of minutes. The channel impulse response functions were measured from the LFM signals in each packet which were separated by $\sim 2 \mathrm{~min}$. We see that the intensity and arrival time of the multipath arrivals vary significantly from packet to packets. This is confirmed by the variation of the arrival time of the peak intensities as a function of transmission time. The temporal variation is apparently caused by the presence of internal waves. 


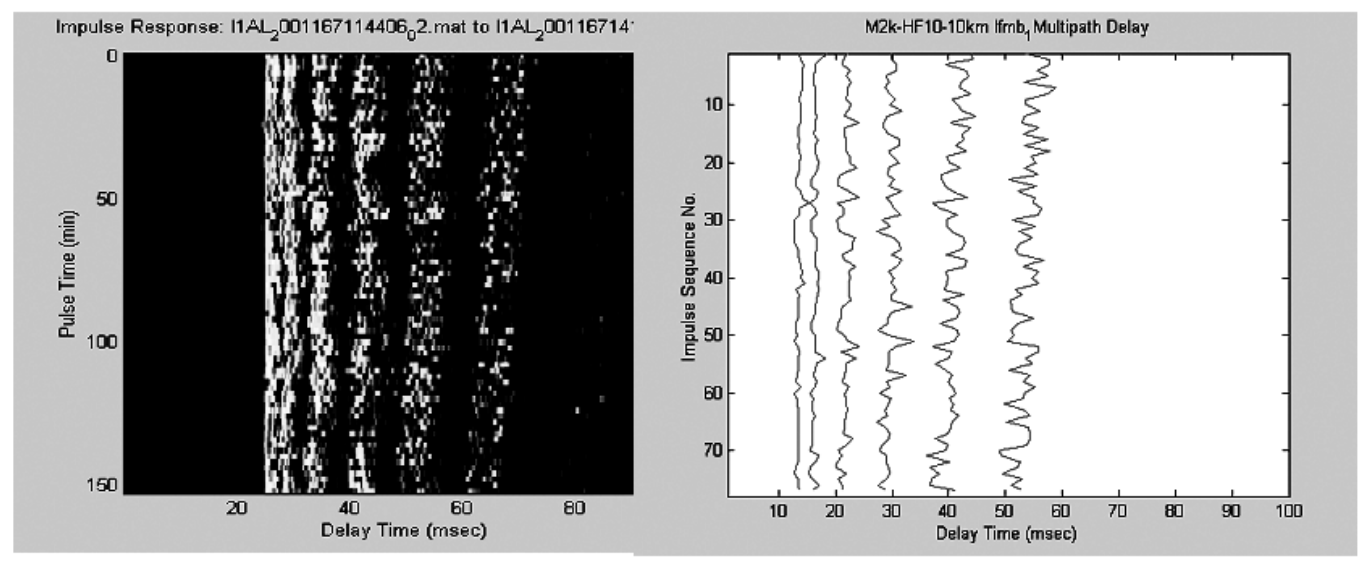

Figure 3. Channel impulse responses functions at the scale of minutes from the ASCOT01 experiment (left). The arrival time of the peak intensities versus the transmission time (right)
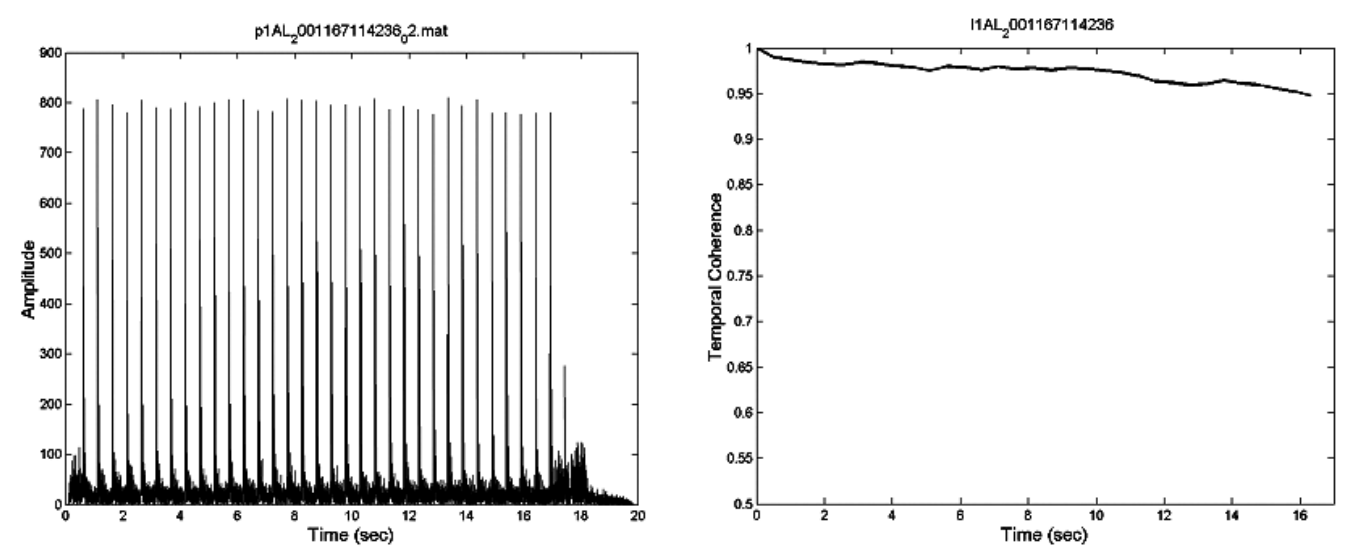

FIGURE 4. Measured channel impulse responses within a packet (left). Temporal coherence of the impulse response functions with respect to the first one (right).

Figure 4 shows the channel impulse response functions measured with consecutive $\mathrm{m}$-sequences within the first packet. It shows little variation of the intensity level as a function of time within the packet. The temporal coherence remains very high $(>0.9)$ as shown in Fig. 4 (right figure). In this case, the DFE works very well - one finds zero BER as shown in Fig. 5. Figure 6 plots the BER and percentage of update for 78 packets transmitted during the two hours time. We see that the BER is $<0.2 \%$ except for one packet. The DFE processor is updated more than 99\% (Fig. 6) even for the packets with zero bit error and high coherence $>0.9$ (e.g. the first packet). If the channel is changing $>99 \%$ of time, the channel could not have such a high coherence value as shown in Fig. 4. It turns out that symbol phase is changing rapidly at the symbol rate (See Ref. 8). The $>99 \%$ update results from the fact that both DFE and PLL attempt to remove the rapid symbol phase change [9]. The conclusion is that at the channel is stable at the scale of tens of seconds (therefore no mystery about the rapid channel change). A different equalizer by applying first passive-phase-conjugation to the data showed that the channel can be equalized with $<5 \%$ update. 
Figure 7 plots the coherence time of the channel as a function of the time. It also plots the output signal-to-noise ratio (SNR) as a function of time. We find a high correlation ( $\sim 0.9$ coherence value) between the channel coherence time and output SNR as shown by the figure on the right. Note that high temporal coherence improves the processor performance in removing ISI and hence a higher output SNR.
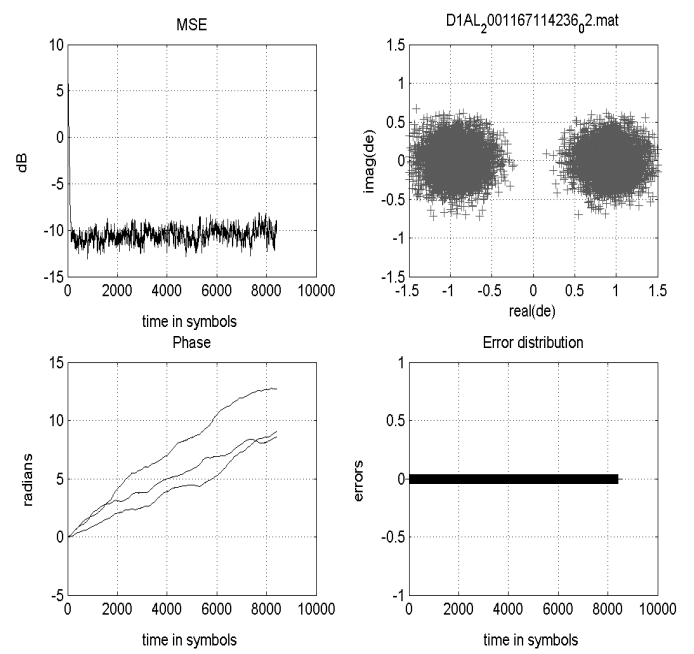

FIGURE 5. Output of the DFE showing the MSE (upper left), symbol phase (lower left), symbol constellations (upper right) and symbol error as a function of symbol number.
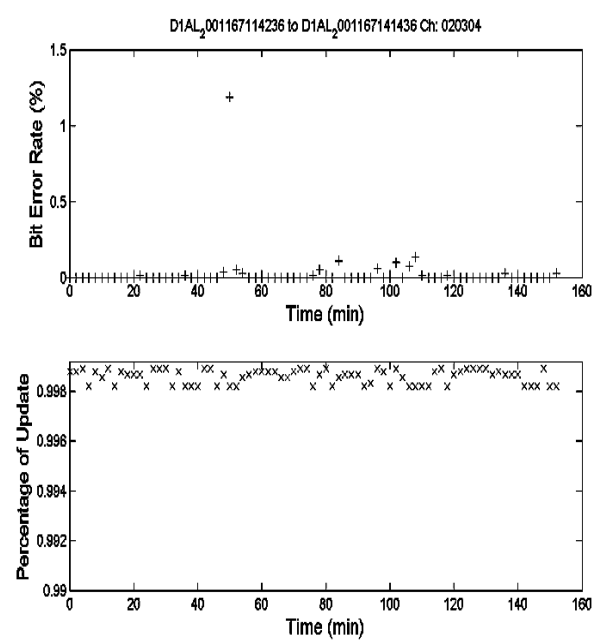

FIGURE 6. BER and percentage of update as a function of transmitted packets displayed by the transmission time.
Coherence Time
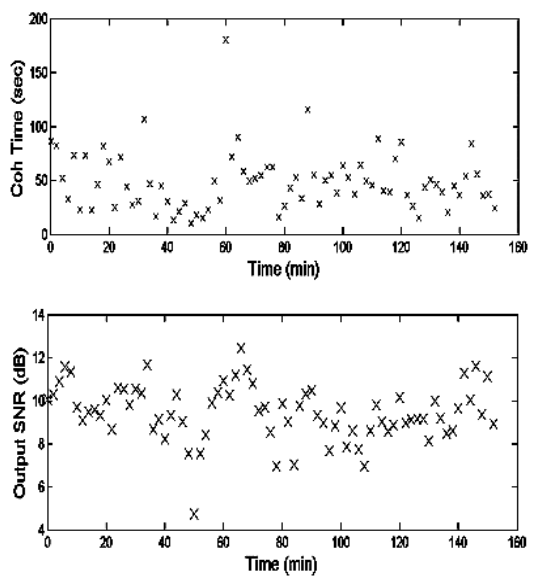

Output SNR
Cross Correlation

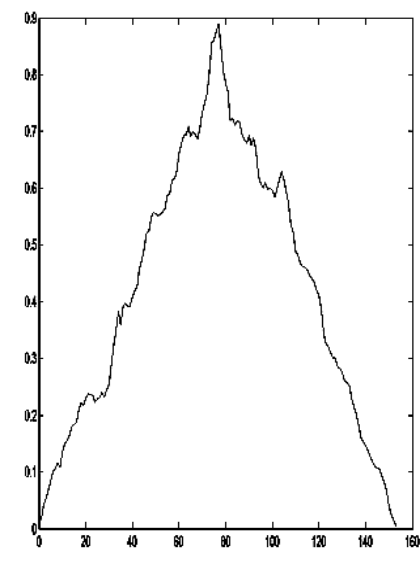

FIGURE 7. The channel coherence time and average output SNR as a function of packet time (left). The normalized cross correlation between coherence time and output SNR (right).

\section{SUMMARY AND DISCUSSIONS}

Temporal coherence of the channel impulse responses plays a vital role for underwater acoustic communications. At mid $(2-5 \mathrm{kHz})$ frequencies, the length of a 
communication packet (5-10 sec for 5-10 kbits) is often shorter than the signal coherence time. The acoustic environment, while randomly changing from packets to packets, presents an instantaneous "deterministic" environment from the point of acoustic communications. In this case, the environmental impact can be mitigated by adequately sampling the channel impulse response function. We use m-sequences to independently verify the temporal variations of the channel within a packet and corroborate with the DFE results.

At low $(<1 \mathrm{kHz})$ frequencies, the packet length for the same amount of data will be long (due to the limited bandwidth) and comparable to the average signal coherence time. The environmental impact is appreciably noticeable by the temporal variations of the impulse responses within a packet (intra-packet). We show in a separate publication [10] that the channel variation can be successfully compensated by a DFE for those (portions of the) packets with high intra-packet coherence.

At high $(>15 \mathrm{kHz})$ frequencies, we note that the average signal coherence time is relatively short. This implies that short packets need to be used to minimize BER.

\section{ACKNOWLEDGEMENT}

This work is supported by the Office of Naval Research. We thank NURC for the opportunities to participate in the Advent99 and ASCOT01 experiments.

\section{REFERENCES}

1. M. Stojanovic, "Recent advances in high-speed underwater acoustic communications," IEEE J. Oceanic Eng. Vol. 21, pp. 125-136, 1996 and references therein.

2. T.C. Yang and M. Siderius, "Temporal coherence and fluctuation of acoustic signals in shallow water," Proc. of the Fifth European Conference on Underwater Acoustics, pp. 63-68. Lyon, France, July 11-14, 2000.

3. T.C. Yang, K. Yoo, and M. Siderius "Internal waves and its effect on signal propagation in the Adventure Bank," Proc. of $8^{\text {th }}$ International Congress On Sound And Vibration, pp.3001-3008, Hong Kong (2 - 6 July, 2001).

4. J. Sellschopp, P. Nielsen and M. Siderius, "Combination of acoustics with high-resolution oceanography," Impact of littoral environmental variability on acoustic prediction and sonar performance, edited by N.G. Pace and F. B. Jensen, Kluwer Academic publishers, pp 19-26 (2002)

5. J. R. Apel et al, "An Overview of the 1995 SWARM Shallow Water Internal Wave Acoustic Scattering Experiment,” IEEE J. Oceanic Eng. 22,465-500 (1997).

6. T. C. Yang, "Temporal fluctuations of broadband channel impulse functions and underwater acoustic communications at 2-5 kHz," Proc. of MTS OCEANS 2002, Vol. 4, pp. 2395-2400, Biloxi (2002).

7. D. Rouseff et al, "Coherence of acoustic modes propagating through shallow water internal waves," J. Acoust. Soc. Am. 111, 1655-1666 (2002).

8. T. C. Yang, "Temporal resolution of time-reversal and passive-phase conjugation for underwater acoustic communications," IEEE J Oceanic Eng. 28, 229-245 (2003).

9. T. C. Yang, "Differences between passive-phase conjugation and decision-feedback equalizer for underwater acoustic communication," IEEE J Oceanic Eng. (2004).

10. T.C. Yang "The effect of internal waves on low frequency underwater acoustic communications," Proc. of International Congress of Acoustics, (2004) 